\title{
Dependência da morfologia dos grãos na textura de cerâmicas ferroelétricas de PBN
}

\section{(Dependence of the grain morphology on texturing of PBN ferroelectric ceramics)}

\author{
F. L. Zabotto, M. Venet, J. A. Eiras, D. Garcia \\ Grupo de Cerâmicas Ferroelétricas, Departamento de Física, Universidade Federal de S. Carlos \\ Rod. Washington Luiz, km 235, S. Carlos, SP 13565-905 \\ g232165@polvo.ufscar.br
}

\begin{abstract}
Resumo
Neste trabalho foram fabricadas cerâmicas ferroelétricas texturadas de niobato de bário e chumbo, puro e dopado com lantânio, utilizando-se a técnica de forjamento a quente com moldes refratários de diferentes formas. Os corpos cerâmicos foram caracterizados nas direções paralelas e perpendiculares ao eixo de prensagem. A análise microestrutural revelou que a dopagem com lantânio aumenta a razão de aspecto dos grãos de PBN, os quais são geralmente obtidos na forma colunar. A maior razão de aspecto possibilitou um maior grau de orientação dos grãos durante o processo de forjamento e, portanto, um aumento de textura do material que se refletiu diretamente nas propriedades físicas macroscópicas.

Palavras-chave: textura, tungstênio-bronze, microestrutura, dopagem, PBN.
\end{abstract}

\begin{abstract}
In this work pure and lanthanum doped textured ferroelectric ceramics of lead-barium niobate (PBN) were produced through hot forging, using different die configurations. The ceramic samples were characterized in the parallel and perpendicular direction respect to the pressing axis. The analysis of the microstructure showed that the increasing of the lanthanum doping increases the aspect ratio of the PBN grains, which generally present a columnar shape. It was concluded that the change of the grain morphology favored a higher grain orientation during the forging process and therefore the enhancing of the material texturing, which was directly reflected in the physical properties of the ceramics.
\end{abstract}

Keywords: texturing, tungsten-bronze, microstructure, PBN, doping.

\section{INTRODUÇÃO}

O niobato de chumbo e bário $\left(\mathrm{Pb}_{\mathrm{x}} \mathrm{Ba}_{1-\mathrm{x}} \mathrm{Nb}_{2} \mathrm{O}_{6}\right.$ ou $\left.\mathrm{PBN}\right)$ é um material ferroelétrico que possui estrutura tungstênio bronze e tem alto potencial para aplicações nas áreas de dispositivos piezoelétricos, eletro-ópticos e fotorefrativos $[1,2]$. Este sistema exibe um contorno de fase morfotrópico em $x \sim 0,63$ (de simetria ortorrômbica para tetragonal), onde a polarização espontânea e outras propriedades ferroelétricas são maximizadas [2-5]. Contudo, o PBN tem sido obtido na forma monocristalina, tendo em vista as dificuldades de se obter material denso na forma cerâmica, já que na síntese se formam pós com partículas alongadas [3].

A textura microestrutural de materiais policristalinos vem sendo estudada há muitos anos, em diferentes sistemas de interesse [6-8], pela vantagem de se obter materiais com propriedades macroscópicas que refletem a anisotropia da rede cristalina. Também em materiais policristalinos ferroelétricos a textura possibilita que as propriedades elétricas, piezoelétricas e mecânicas, sejam anisotrópicas [911]. Como a anisotropia cristalina é perdida com o padrão aleatório de grãos, apenas a textura garante às cerâmicas o acesso às propriedades favoráveis dos monocristais.

Os métodos mais comuns para se obter textura são: extrusão, forjamento ou prensagem a quente e colagem de fita [9]. Em tais processos a textura ocorre somente devido à morfologia dos grãos, os quais devem ser não-equiaxiais de tal forma a se orientarem e crescerem preferencialmente em uma direção. Materiais ferroelétricos policristalinos com estrutura tungstênio-bronze e simetria tetragonal, geralmente, apresentam grãos alongados e com hábito de crescimento na direção [001] (direção de polarização) ao longo do eixo longitudinal, como no caso do sistema niobato de estrôncio e bário [12]. Portanto, as técnicas de textura são aplicáveis nestes materiais para a obtenção de anisotropia das propriedades, auxiliando inclusive na densificação. 
Neste trabalho, o efeito da morfologia (razão de aspecto) dos grãos foi analisado no processo de obtenção de textura em cerâmicas ferroelétricas do sistema $\mathrm{Pb}_{\mathrm{x}} \mathrm{Ba}_{1-\mathrm{x}} \mathrm{Nb}_{2} \mathrm{O}_{6}$, com $\mathrm{x}=0,56$, dopado com lantânio, utilizando a técnica de forjamento a quente.

\section{EXPERIMENTAL}

Os pós de $\mathrm{Pb}_{0,56} \mathrm{Ba}_{0,44} \mathrm{Nb}_{2} \mathrm{O}_{6}+\mathrm{x} \%$ em peso de $\mathrm{La}_{2} \mathrm{O}_{3}$ (ou PBN56 + x\% de $\mathrm{La}_{2} \mathrm{O}_{3}$ ), com x =0,0, 0,5, 1,0, 1,5, 2,0 e 4,0, foram preparados pelo método convencional de mistura de óxidos. Os reagentes de partida, $\mathrm{PbO}, \mathrm{Ba}\left(\mathrm{NO}_{3}\right)_{2}, \mathrm{Nb}_{2} \mathrm{O}_{5}$ e $\mathrm{La}_{2} \mathrm{O}_{3}$ (todos com pureza analítica), foram misturados em frascos de polietileno contendo álcool isopropílico e cilindros de zircônia estabilizada, durante 24 h. Após a secagem em estufa a vácuo, os pós foram calcinados a $1240{ }^{\circ} \mathrm{C}$, por $3 \mathrm{~h}$, e conformados a frio, utilizando-se matriz de aço (cilíndrica ou retangular) em uma prensa uniaxial com pressão de $250 \mathrm{MPa}$. Subseqüente prensagem isostática a frio foi realizada para redução de gradientes de densidade dos corpos cerâmicos. Um lote de amostras (na forma de discos) foi sinterizado em fornos tipo mufla, em $1270{ }^{\circ} \mathrm{C} / 2 \mathrm{~h}$, para a análise das características estruturais e microestruturais do material em função da concentração de lantânio. Um estudo de otimização da atmosfera de sinterização, não apresentado aqui, mostrou que não há a necessidade de atmosfera rica em óxido de chumbo durante a queima, para a obtenção de corpos cerâmicos relativamente densos e com a fase esperada. Como discutido a seguir, os resultados apresentados pelo material sinterizado convencionalmente permitiu a escolha das composições aparentemente mais adequadas para o estudo do efeito da morfologia dos grãos na textura das cerâmicas de PBN. Sendo assim, amostras (obtidas por prensagem uniaxial e isostática a frio na forma cilíndrica e/retangular), nas composições escolhidas, foram densificadas/forjadas por prensagem a quente, a $1270^{\circ} \mathrm{C} / 2$ $\mathrm{h}$, em atmosfera de oxigênio, utilizando-se uma prensa uniaxial Thermal Inc. (modelo HP22-0614-SC). Testou-se dois moldes refratários em etapas diferentes: um cilíndrico, que permitia o forjamento livre do material no plano perpendicular ao eixo de prensagem (Fig. 1a), e outro retangular, cujo forjamento era livre em apenas uma das direções no plano perpendicular ao eixo de
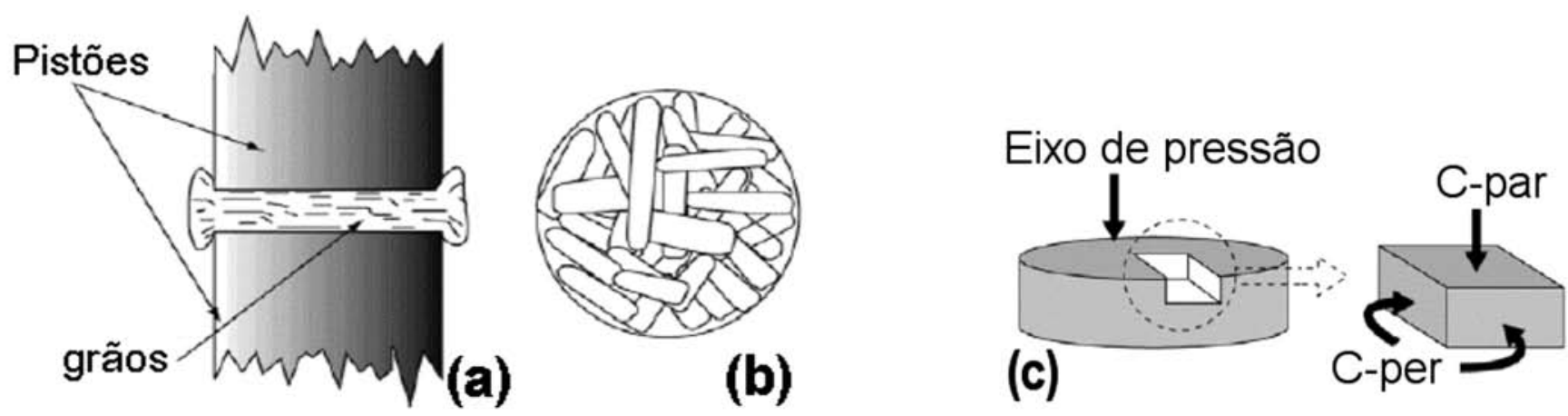

Figura 1: (a) Representação esquemática do molde cilíndrico utilizado durante o forjamento a quente; (b) esquema do arranjo dos grãos no plano perpendicular ao eixo de prensagem, para este caso; (c) amostra extraída para análise e denominação de seus planos de corte, para este caso.

[Figure 1: (a) Schematic representation of the cylindrical die used for the hot forging; (b) Scheme of the grain arrange in the perpendicular plane to the pressing axis, for this case; (c) Extract sample for the analysis and denomination of its cut plane, for this case.]
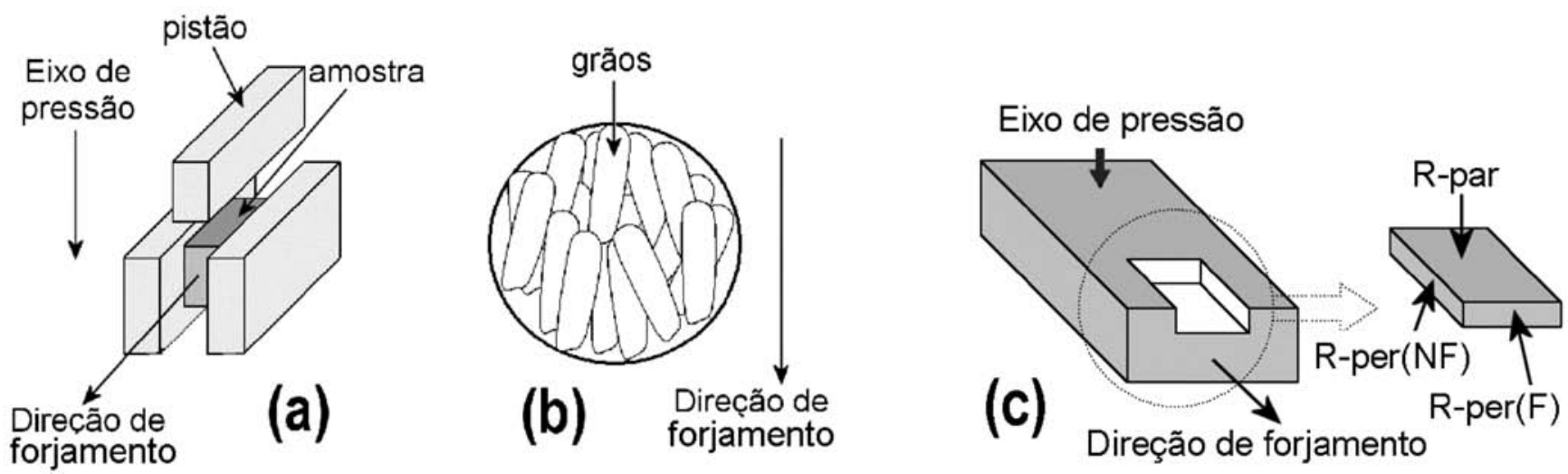

Figura 2: (a) Representação esquemática do molde retangular utilizado durante o forjamento a quente; (b) esquema do arranjo dos grãos no plano perpendicular ao eixo de prensagem, para este caso; (c) amostra extraída para análise e denominação de seus planos de corte, para este caso. [Figure 2: (a) Schematic representation of the rectangular die used for the hot forging; (b) Scheme of the grain arrange in the perpendicular plane to the pressing axis, for this case; (c) Extract sample for the analysis and denomination of its cut plane, for this case.] 
prensagem (Fig. 2a). As Figs. 1b e 2b representam os arranjos de grãos esperados para cada caso, respectivamente, vistos de um plano perpendicular ao eixo da pressão aplicada durante o forjamento. Amostras foram extraídas das cerâmicas forjadas, como representado nas Figs. 1c e 2c. Nestas figuras estão indicadas as denominações de cada tipo de amostra segundo as diferentes direções dos planos de corte em relação à direção de pressão.

A análise por difração de raios $X$ do material foi realizada utilizando-se um difratômetro Rigaku, de ânodo rotatório, radiação Cuk ${ }_{\alpha}$, com $2 \theta$ entre 10 e $60^{\circ}$, do Grupo de Cristalografia do IFSC/USP. A análise microestrutural foi feita mediante a técnica de microscopia eletrônica de varredura (MEV), com um microscópio Jeol 5400 LV.

Utilizando-se um sistema automatizado para aquecimento/ resfriamento da amostra, acoplado a um analisador de impedância Hewlett Packard-4194A, realizou-se as medidas para a obtenção da permissividade elétrica, $\varepsilon$ ', em função da temperatura (e da freqüência, embora apenas os resultados em $1 \mathrm{kHz}$ estejam sendo apresentados). Para tanto, nas faces das amostras, definidas pelos planos de corte apresentados nas Figs. 1c e 2c, foram depositados eletrodos de platina por "sputtering". As medidas foram realizadas durante o resfriamento, com taxa de $2{ }^{\circ} \mathrm{C} / \mathrm{min}$.

\section{RESULTADOS E DISCUSSÃO}

Sinterização convencional de cerâmicas de PBN56 + $x \%$ de $\mathrm{La}_{2} \mathrm{O}_{3}$

Observou-se, pela análise por difração de raios X (cujos difratogramas não estão apresentados aqui), que o material sinterizado apresenta apenas a fase tungstênio-bronze (TB), com simetria indistinta da tetragonal (4 mm), no caso de todas as composições com exceção da com $4 \%$ em peso de óxido de lantânio, que mostra a coexistência das fases PBN TB com simetria tetragonal paraelétrica (4/mmm) e $\mathrm{PbO}$ (massicot).

As micrografias de superfície das cerâmicas de PBN56 $+\mathrm{x} \%$ de $\mathrm{La}_{2} \mathrm{O}_{3}$, sinterizadas convencionalmente, com $\mathrm{x}=0,0$; $x=0,5 ; x=1,0 ; x=2,0$ e $x=4,0$, podem ser observadas na Fig. 3 . Percebe-se que os grãos têm a forma colunar, típica de cerâmicas com estrutura tungstênio-bronze [12], e que estão distribuídos aleatoriamente. As análises por difração de raios X e impedância de cortes diferentes destas amostras não mostram características anisotrópicas. Um fator interessante da microestrutura, no entanto, é que, com o aumento da concentração de lantânio, a razão de aspecto dos grãos aumenta com o incremento do dopante, variando de 2:1, para a cerâmica de PBN56 puro, para até 6:1 no caso da composição com $4 \%$ em peso de $\mathrm{La}_{2} \mathrm{O}_{3}$. A substituição do íon $\mathrm{La}^{3+}$ nos sítios catiônicos $\left(\mathrm{Ba}^{2+}\right.$ e/ou $\left.\mathrm{Pb}^{2+}\right)$ pode estar aumentando a difusão atômica, pela introdução de vacâncias para a manutenção da eletro-neutralidade, favorecendo o hábito de crescimento nos planos ao longo do grão. Como uma alta razão de aspecto é extremamente favorável para fins de textura, já que grãos longos podem ser mais bem orientados durante o forjamento do material, este resultado sugere que as cerâmicas com maior concentração de lantânio devem atingir um grau de textura microestrutural e/ou cristalográfica maior. Portanto, para fins de comparação do efeito da morfologia do grão na textura final das amostras, as composições $x=0,0, x=2,0$ e $\mathrm{x}=4,0$ foram escolhidas para a etapa de forjamento a quente $\mathrm{e}$ para a subseqüente caracterização microestrutural e dielétrica, como discutido a seguir.

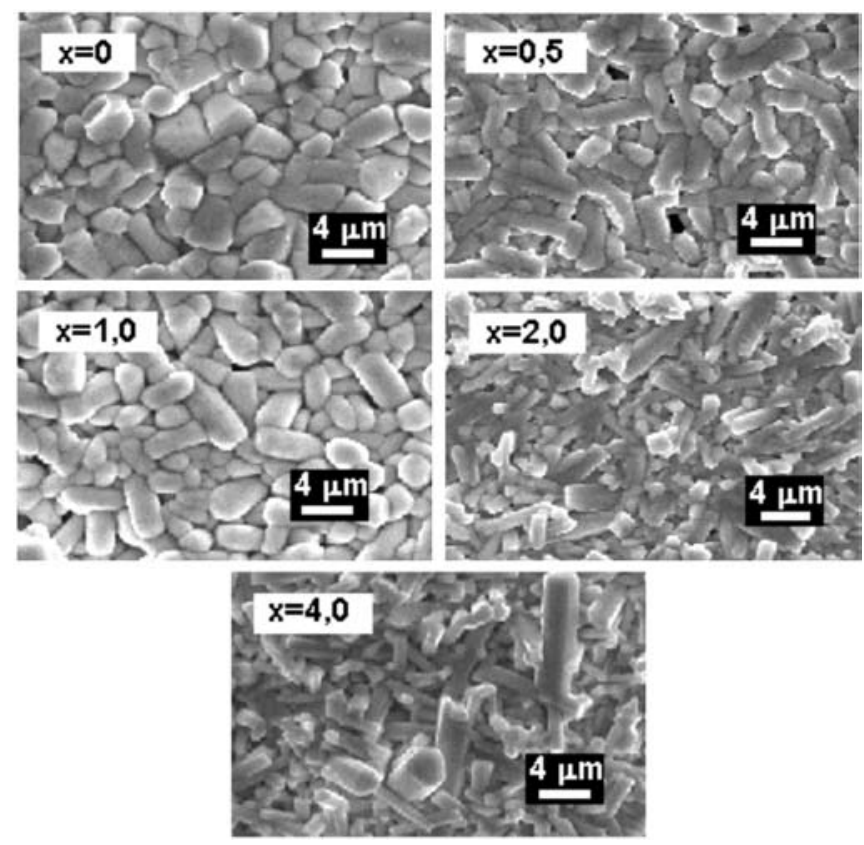

Figura 3: Micrografias de superfície das cerâmicas de PBN56 + $\mathrm{x} \%$ peso de $\mathrm{La}_{2} \mathrm{O}_{3}$, como sinterizadas (em forno tipo mufla, a $1270{ }^{\circ} \mathrm{C}$, por $2 \mathrm{~h}$, no ar)

[Figure 3: Surface micrographs of the PBN56+xwt\% of $\mathrm{La}_{2} \mathrm{O}_{3}$ ceramics, as sintered (in muffle type furnace, at $1270{ }^{\circ} \mathrm{C}$ for $2 \mathrm{~h}$, in air).]

Cerâmicas de $\mathrm{PBN} 56+x \%$ de $\mathrm{La}_{2} \mathrm{O}_{3}$ forjadas a quente com molde cilíndrico

A Fig. 4 ilustra o comportamento da permissividade elétrica em função da temperatura, para as cerâmicas de PBN56 + x\% de $\mathrm{La}_{2} \mathrm{O}_{3}$, com x=0,0, 2,0 e 4,0, forjadas a quente com o molde refratário cilíndrico, no caso dos cortes cujas direções do plano são paralela e perpendicular à direção de prensagem (C-par e Cper, respectivamente). Verifica-se anisotropia das propriedades dielétricas, principalmente quando se compara os valores da permissividade elétrica, medida na direção perpendicular e paralela à direção de pressão, em temperaturas próximas a temperatura de transição de fase ferro-paraelétrica. No entanto, as cerâmicas dopadas com lantânio, especialmente a com x= 2,0, apresentam maior grau de anisotropia do que a de PBN puro. Outras características da influência do $\mathrm{La}^{3+}$ devem ser cuidadosamente analisadas como, por exemplo, o aumento do caráter difuso da transição (picos mais largos), e a diminuição da 
temperatura de transição ferro-paraelétrica que, para a cerâmica dopada com $4 \%$ de $\mathrm{La}_{2} \mathrm{O}_{3}$, foi de aproximadamente $-50{ }^{\circ} \mathrm{C}$ (já esperado pelo resultado de DRX à temperatura ambiente da amostra obtida convencionalmente, em que se observou a fase tetragonal paraelétrica para esta composição). O favorecimento de grãos mais alongados, com razão de aspecto maior, devido à adição de lantânio ao PBN, proporcionou maior grau de textura microestrutural/cristalográfica no material, refletindo-se nas características dielétricas. Contudo, no caso da composição com $x=4,0$, efeitos concorrentes fazem com que a anisotropia dielétrica seja menos efetiva do que a com $\mathrm{x}=2,0$. Embora a textura microestrutural, que induz à textura cristalográfica, seja favorecida devido a maior razão de aspecto do grão da $x=4,0$, a anisotropia estrutural local (da cela unitária, 4/mmm) é menor.
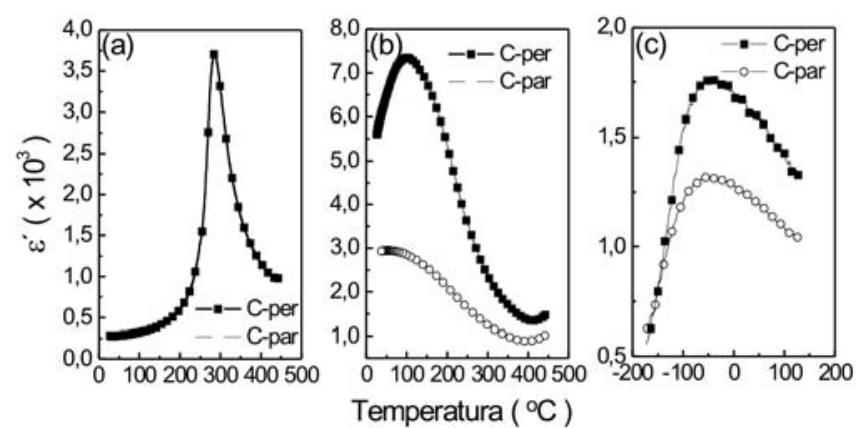

Figura 4: Permissividade elétrica, $\varepsilon$ ', em função da temperatura, para as cerâmicas de $\mathrm{PBN} 56+\mathrm{x} \%$ de $\mathrm{La}_{2} \mathrm{O}_{3} \operatorname{com}$ : (a) $\mathrm{x}=0$, (b) $\mathrm{x}=2,0 \mathrm{e}$ (c) $\mathrm{x}=4,0$ forjadas a quente com o molde refratário cilíndrico. C-per e C-par referem-se aos planos de cortes com direção perpendicular e paralela à de prensagem, respectivamente, representados nas figuras $1 \mathrm{c}$ e $2 \mathrm{c}$.

[Figure 4: Relative permittivity, $\varepsilon$ ', as a function of the temperature, for the PBN56+xwt\% of $\mathrm{La}_{2} \mathrm{O}_{3}$ ceramics with: (a) $x=0$; (b) $x=2.0$ and (c) $x=4.0$; which were hot forged with the cylindrical refractory die. $C$ per and C-par refer to the cut planes with perpendicular and parallel direction, respectively, which are represented in Figures 1c and 2c.]

Na Fig. 5, onde se encontram os perfis de difração de raios $\mathrm{X}$ das amostras forjadas a quente, observa-se que a composição PBN56 com $x=0,0$ não apresenta mudanças significativas entre as diferentes direções de medida, indicando baixo grau de textura cristalográfica. $\mathrm{O}$ mesmo ocorreu para o material $\mathrm{x}=$ 4,0 (não apresentado aqui). No entanto, para a composição $x=$ 2,0 se vê um aumento significativo da intensidade das reflexões pertencentes aos planos (001) e (002) na direção perpendicular ao eixo de pressão (C-per), indicando que maior quantidade desses planos pode ser encontrada nessa direção.

Cerâmicas de $\mathrm{PBN} 56+x \%$ de $\mathrm{La}_{2} \mathrm{O}_{3}$ forjadas a quente com molde retangular

A técnica de forjamento a quente com o molde retangular foi aplicada para as composições PBN56+x\% $\mathrm{La}_{2} \mathrm{O}_{3}$, com x=0,0 e 2,0, objetivando-se a otimização do processo de textura e a melhor análise do efeito da morfologia (razão de aspecto) dos grãos.

A Fig. 6 mostra a superfície de fratura das cerâmicas de
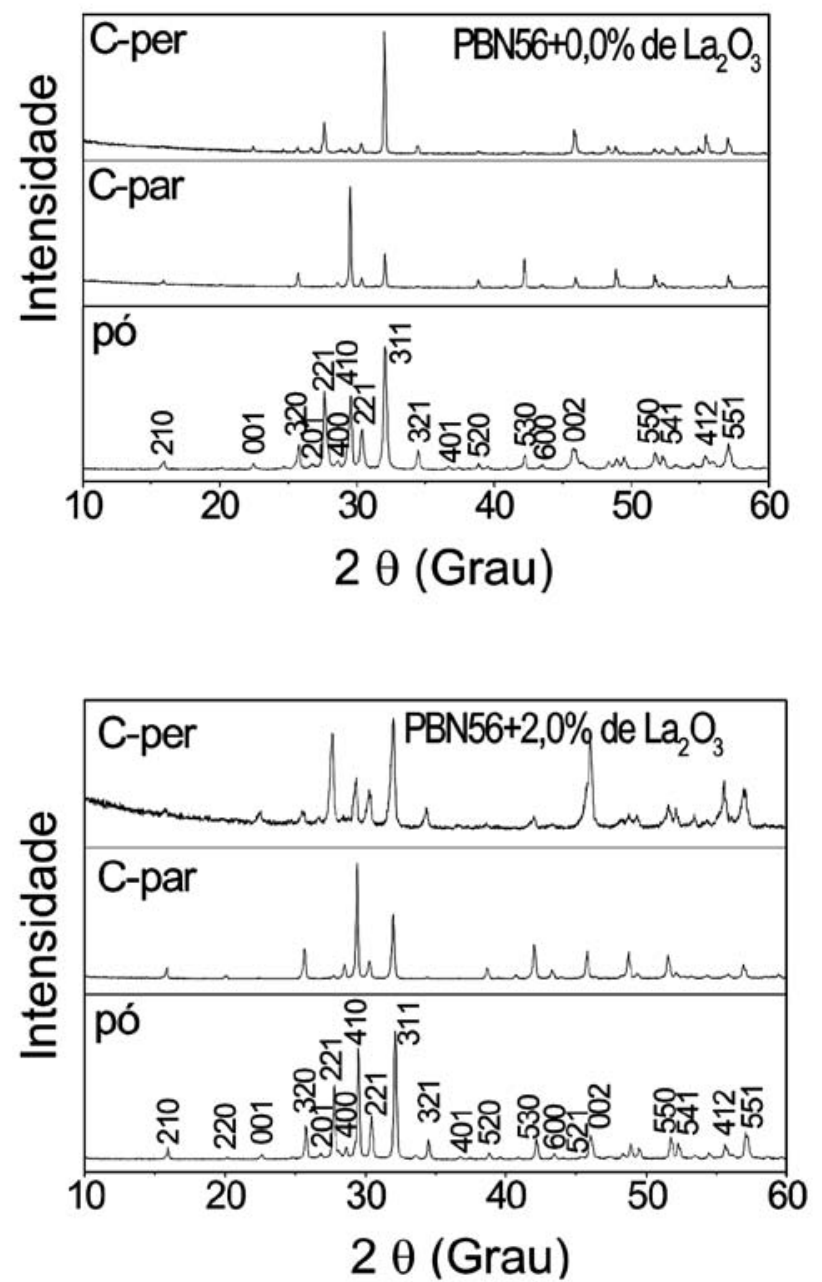

Figura 5: Perfis de difração de raios $X$, à temperatura ambiente, para cerâmicas forjadas a quente (molde cilíndrico) de PBN56+x\% de $\mathrm{La}_{2} \mathrm{O}_{3}$, com $\mathrm{x}=0,0$ e 2,0, nos casos do material na forma de pó e nos cortes C-par e C-per.

[Figure 5: Room temperature $X$-ray diffraction patterns of the hotforged $\mathrm{PBN} 56+x w t \%$ of $\mathrm{La}_{2} \mathrm{O}_{3}$ ceramic (cylindrical die), with $x=0$ and 2.0, in the cases for the powder material and for the C-par and C-per cuts.]

PBN56, com $\mathrm{x}=0,0$ e $\mathrm{x}=2,0$ de $\mathrm{La}_{2} \mathrm{O}_{3}$, forjadas a quente com o molde refratário retangular, para diferentes planos de corte. Observa-se que a cerâmica de PBN56 pura, não apresenta grandes diferenças entre as direções perpendiculares ao eixo de pressão, exceto no caso de alguns grãos que apresentam crescimento anormal ao longo da direção de forjamento. Por sua vez, a cerâmica de PBN dopada com $\mathrm{La}^{3+}$ apresenta um alto nível de orientação dos grãos com hábito de crescimento ao longo da direção de forjamento.

As curvas de permissividade elétrica em função da temperatura para as amostras da composição $\mathrm{x}=0,0$ (Fig. 7) mostram que, na direção de pressão (R-par) e na direção perpendicular à pressão em que não ocorreu forjamento (R- 

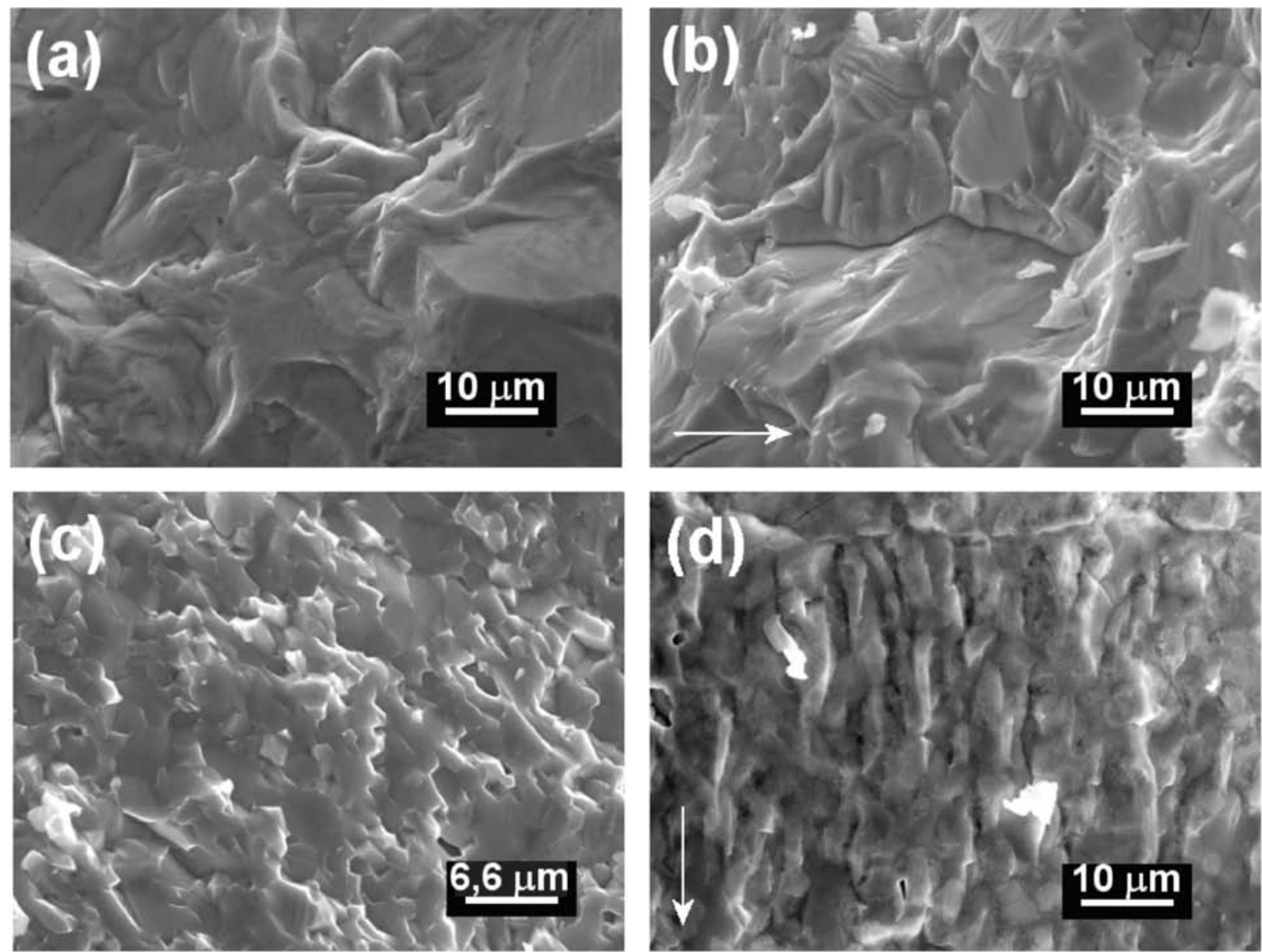

Figura 6: Superfície de fratura das cerâmicas de PBN56+x\% de $\mathrm{La}_{2} \mathrm{O}_{3}$ : (a) x=0,0; R-per(F); (b) x=0,0, R-per(NF); (c) x=2,0, R-per(F); (d) $\mathrm{x}=2,0$, R-per(NF). As setas indicam a direção de forjamento.

[Figure 6: Fracture surface of the PBN56+xwt\% of L $a_{2} O_{3}$ ceramics: (a) $x=0 ; R-p e r(F) ;(b) x=0, R-p e r(N F) ;(c) x=2.0, R-p e r(F) ;(d) x=2.0$, $R$-per(NF). The arrows indicate the forging direction.]

per(NF)), a resposta dielétrica é equivalente, sendo um pouco diferentes daquela na direção do forjamento (R-per(F)). Isto indica que os grãos cresceram orientados somente na direção de forjamento da cerâmica como esperado para o molde retangular, embora a textura não tenha sido mais efetiva do que aquela obtida com o molde cilíndrico. Outro aspecto a levar em consideração é o aumento do valor absoluto da permissividade elétrica desta cerâmica relativamente àquela forjada no molde circular. Este resultado acompanha a maior densificação do material. $\mathrm{Na}$ composição $\mathrm{x}=2,0$, ocorreu uma maximização de $\varepsilon$ ' tanto para a direção R-per(F) quanto R-per(NF) (Fig. 7). Neurgaonkar e colaboradores [4] observaram que a adição de $\mathrm{La}^{3+}$ em composições de PBN com simetria tetragonal, mas próximas ao contorno de fase morfotrópico, pode induzir a ocorrência de fase TB de simetria ortorrômbica. Em monocristais de PBN, com simetria ortorrômbica, a maior resposta dielétrica e ferroelétrica ocorre na direção [010] [3]. Uma resposta intermediária é observada na direção [100] e, a menor, na direção [001]. Por outro lado, o hábito de crescimento dos grãos de PBN, na fase ortorrômbica, pode ser na direção [100] e [110] ao longo do comprimento do grão [3]. Desta forma, se considerada a direção [100] como a de crescimento do grão e, portanto, a da direção de forjamento da cerâmica (ou seja, R-per(F)), a permissividade elétrica nesta direção deve ser maximizada, como observado. Sendo assim, a outra direção perpendicular ao eixo de pressão (R-per(NF)), deve ser das direções cristalográficas [010] e [100], que também apresentaria alto valor de permissividade elétrica, como observado. Desta forma, pode-se deduzir que a dopagem de lantânio tenha favorecido a fase ortorrômbica m2m (ou pseudo-tetragonal já que não foi detectada pela análise de DRX realizada), além de modificar a morfologia dos grãos de cerâmicas de PBN. A anisotropia observada para o material dopado, portanto, pode ter sido maior por causa da simetria da cela unitária que passou a ser $\mathrm{m} 2 \mathrm{~m}$. Para o esclarecimento desta questão, o material puro $(x=0,0)$ de simetria tetragonal $4 \mathrm{~mm}$, foi forjado a quente com o molde retangular, a partir de pó não-moído com partículas com comprimento de até $5 \mu \mathrm{m}$. As curvas de permissividade elétrica em função da 


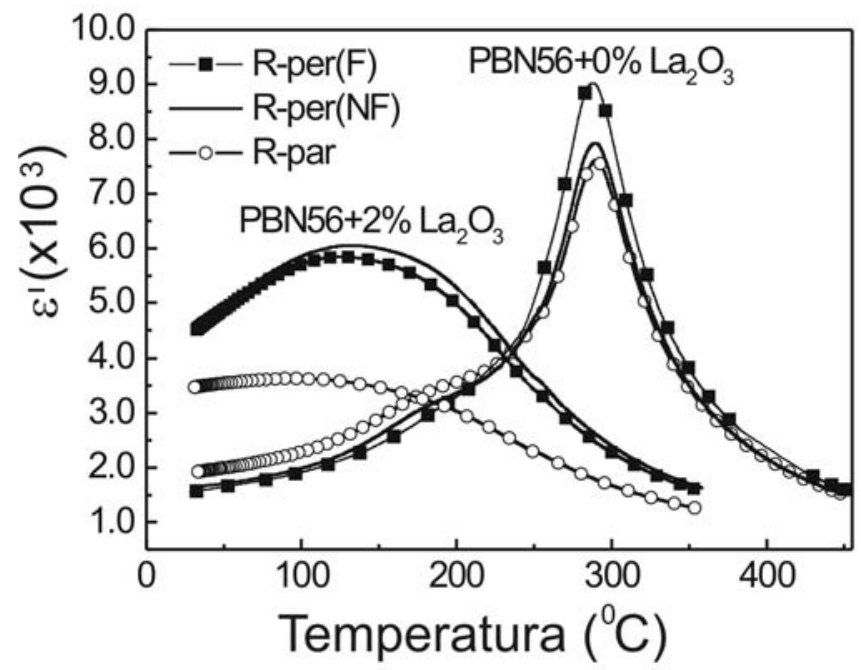

Figura 7: Permissividade elétrica em função da temperatura, das cerâmicas de $\mathrm{PBN} 56+\mathrm{x} \%$ de $\mathrm{La}_{2} \mathrm{O}_{3}$ forjadas a quente com o molde retangular, para as diferentes direções de corte.

[Figure 7: Electric permittivity as a function of the temperature (in the different cut directions) for the PBN56+xwt\% of $\mathrm{La}_{2} \mathrm{O}_{3}$ ceramics, which were hot forged using the rectangular die.]

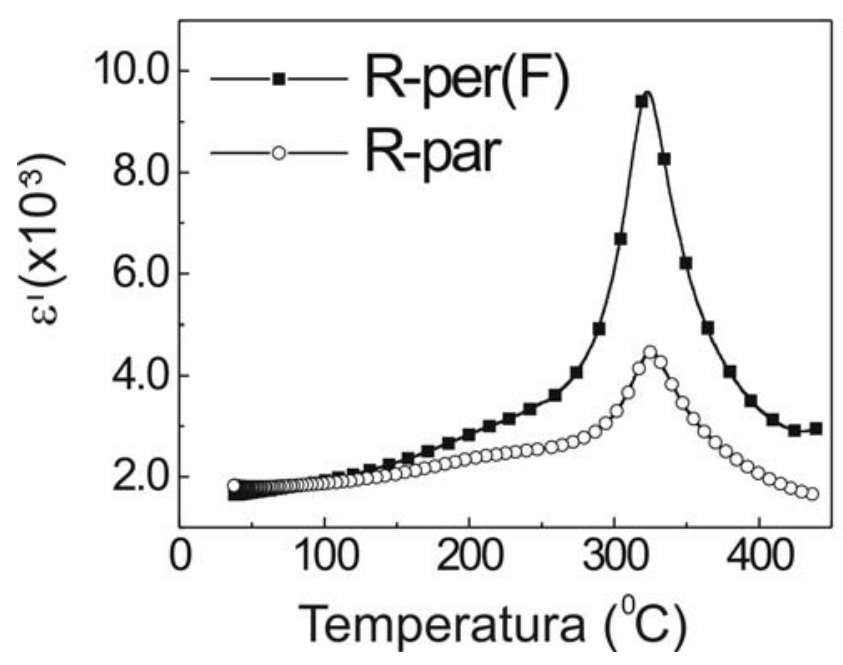

Figura 8: Permissividade elétrica em função da temperatura, da cerâmica de PBN56, com $x=0,0$, forjada a quente com o molde retangular a partir de pó não moído e medida em diferentes direções.

[Figure 8: Electric permittivity as a function of the temperature (in the different cut directions) for the PBN56 ceramic, with $x=0$, hot forged starting from not milled powders and using the rectangular die.]

temperatura, para a cerâmica de PBN56, $\operatorname{com} x=0,0$, forjada a quente com o molde retangular a partir de pó não moído, são apresentadas na Fig. 8. O grau de anisotropia dielétrica alcançado nesta cerâmica é bem maior do que aquele obtido na cerâmica sinterizada a partir de pó moído, confirmando que o efeito da morfologia do grão na textura do PBN predomina sobre o da simetria.

\section{CONCLUSÕES}

A dopagem com lantânio de cerâmicas de PBN56 favorece a formação de grãos alongados com hábito de crescimento na direção [100] (para simetria m2m) e com alta razão de aspecto, quando comparada ao do material puro sintetizado sob as mesmas condições. Por causa da morfologia das partículas, as cerâmicas dopadas podem apresentar maior grau de textura microestrutural/cristalográfica quando o processamento adequado para a orientação dos grãos é aplicado. Por outro lado, o lantânio diminui a temperatura de transição de fase ferro-paraelétrica, aumentando a simetria da cela unitária em faixas mais largas de temperatura, que compete negativamente para a textura do material. Há, portanto, uma concentração intermediária deste dopante em que a textura e anisotropia de propriedades podem ser maximizadas. Caso a razão de aspecto dos grãos seja alta, para uma fase de baixa simetria local, o material poderá apresentar um alto grau de textura. O procedimento de forjamento uniaxial a quente (molde retangular), neste caso, mostra-se como um dos mais adequados para a obtenção de cerâmicas de PBN com características anisotrópicas.

\section{AGRADECIMENTOS}

Os autores agradecem CNPq e FAPESP pelo apoio financeiro e ao Sr. Francisco J. Picon pelo apoio técnico.

\section{REFERÊNCIAS}

[1] M. Yokosuka, Jpn. J. Appl. Phys. 16 (1977) 379.

[2] J. R. Oliver, R. Ratnakar, R. R. Neurgaonkar, L. E. Cross, J. Am. Ceram. Soc. 72 (1989) 202.

[3] R. Guo, Ferroelectric Properties of Lead Barium Niobate Compositions Near the Morphotropic Phase Boundary, Tese de Doutorado, Pennsylvania State University, PA, USA (1990).

[4] R. R. Neurgaonkar, J. R. Oliver, J. G. Nelson, L. E. Cross, Mater. Res. Bull. 26 (1991) 771.

[5] I. A. Santos, D. Garcia, J. A. Eiras, J. Appl. Phys. 93 (2003) 1701.

[6] D. Brandon, D. Chen, H. Chan, Mater. Sci. Eng. A 195 (1995) 189.

[7] Z. J. Shen, Z. Zhao, H. Peng, M. Nygren, Nature 417 (2002) 266.

[8] Y. Saito, H. Takao, T. Tani, T. Nonoyama, K. Takatori, T. Homma, T. Nagaya, M. Nakamura, Nature 432 (2004) 84.

[9] E. M. Sabolsky, A. R. James, S. Kwon, S. T. McKinstry, G. L. Messing, Appl. Phys. Lett. 78 (2001) 2551.

[10] J. Ricote, L. Pardo, A. Moure, A. Castro, P. Millán, D. Chateigner, J. Eur. Ceram. Soc. 21 (2001) 1403

[11] M. Venet, A. Vendramini, I. A. Santos, J. A. Eiras, D. Garcia, Mater. Sci. Eng. B 117 (2005) 254.

[12] S. Nishiwaki, J. Takahashi, K. Kodaira, M. Kishi, J. Appl. Phys. Jpn. 35 (1996) 5137.

(Rec. 20/07/2005, Ac. 25/08/2005) 